\title{
Prevalence of Neisseria Gonorrhoeae in HIV Patients Attending Federal Teaching Hospital Abakaliki, South-Eastern Nigeria
}

\author{
Nwele, David E. \\ Department of Applied Biology Faculty of Biological Science Ebonyi State University, Abakaliki Nigeria.
}

\begin{abstract}
A survey on this study, was carried out to investigate the prevalence of Neisseria gonorrhoeae in HIV patients attending Federal Teaching Hospital, Abakaliki. A total of 60 patients were examined and the result showed that 40 patients $(66.7 \%)$, were co-infected with Neisseria gonorrhoeae. Individuals within the active age group (21-30) recorded the highest occurrence of infection. The prevalence among sexes revealed that females had the highest prevalence (40\%) compared to their male counterparts (26.7\%). Marital status of the infected patients was also observed to play a role in the prevalence of the infection, with single unmarried individuals at the highest risk of infection. Testing individuals who contract STDs or HIV will help reduce the risk of transmission since infection with one could pose a risk for the other
\end{abstract}

Key Word: Prevalence, Neisseria Gonorrhoeae, HIV Patients, Abakaliki, Nigeria.

\section{Introduction}

Neisseria gonorrhoeae is a species of gram-negative coffee bean-shaped diplococcic bacteria which causes a disease known as Gonorrhoeae, a sexually transmitted diseases (Ryam, 2004). The disease may be transmitted by vaginal, cervix, rectum, pharynx (throat), or eyes. In men, Neisseria gonorrhoeae infects urethra and is usually with symptoms, causing dysuria and acute urethritis with a purulent discharge. Among women, Neisseria gonorrhoeae infects the cervix, urethra, vulva and rectum. Rectal infections occur in up to $40 \%$ of women infected with gonorrhoeae. Generally, in about $95 \%$ of patients, gonococci can be found in urethral discharge or urine sediments. The infection may spread to prostrate, bladder, and epididymes, causing inflammation and swollen epididymes and may lead to sterility (Cheesbrough, 2006).Symptoms which typically appear 2-14 days after exposure include pain or burning during urination or defecation; a yellowish or greenish (pus-like) penicle, vaginal, cervical or rectal discharge; swelling and tenderness of the vulva, sore throat, and a false urge to urinate or defecate. An estimated $10 \%$ of men and up to $80 \%$ of women are asymptomatic.Gonorrhoeae is diagnosed using a gram stain, in which a sample of genital fluid is cultured, stained and examined under a microscope; a urine test for gonorrhoeae is also available. Untreated gonorrhoeae can lead to pelvic inflammatory diseases (PID) or epididymitis, as well as damage to the joints. In pregnant women, gonorrhoeae is associated with premature labour, miscarriage and stillbirth, and it may be transmitted to an infant during birth (CDC, 1998). Sexually transmitted diseases (STDs) such as gonorrhoeae are associated in several ways with HIV, because STDs and HIV are spread by similar types of sexual activity. People who engage in behaviours that transmit HIV are also more likely to contract STDs (Wasserheit, 1992).person who contract an STDs may also have put themselves at risk for HIV and should be tested. Likewise, a person who contract HIV should be tested for other STDs. In addition to epidemiological pattern of HIV and STD transmission, immune damage related to HIV infection can influence the progression of STDs and vice-versa.

\section{1 study area/population}

\section{Materials And Methods}

The study area is federal teaching hospital Abakaliki, located in the capital city of Ebonyi State SouthEstern Nigeria.The study population comprises about $60 \mathrm{HIV}$ patients that attend federal Teaching Hospital Abakaliki. The study participants were well informed about the research which they freely consented.

\section{2 sample collection}

A total of 60 urine samples ( $5 \mathrm{ml}$ each) were collected from each of the study participants using a sterile bottle. The sample bottles were clearly labeled using information such as age, sex, occupation and level of education obtained from the participants. All the urine samples collected were allowed to stand for about 2 hours to allow sedimentation to occur after which they were centrifuge. The supernatants were discarded while the sediments were stored at $20^{\circ} \mathrm{c}$ until ready to use. 


\section{3 media preparation}

The chocolate agar medium used was prepared according to the manufacturer's directives. $16.8 \mathrm{~g}$ of the media was dissolved in a litre of distilled water and boiled in order to dissolve. The agar sterility was performed by autoclaving for $15 \mathrm{mins}$ at $121^{\circ} \mathrm{c}$. It was allowed to cool for $5 \mathrm{mins}$ before dispensing into sterile petri dishes and later allowed to gel before storing. Sterility was checked by absence of growth after 24hours. It was later stored in the refrigerator pending when it will be used.

\section{4 inoculation of specimen}

The urine samples were respectively inoculated into chocolate agar medium and incubated at $37^{\circ} \mathrm{c}$ and checked at intervals of 24-48hrs to obtain colonies. A small quantity of the colony of Neissria gonorrhoeae was collected using sterile wire loop. It was then streaked on nutrient agar as described by Okereke and Kanu, (2004) to obtain a pure culture.

\section{5 gram staining /mounting}

A smear of a day old culture was made on a clear, grease-free slide and air dried by passing it over a Bunsen burner flame for a while as described by (Cheesbrough, 2006). The heat fixed smear was stained with crystal violet stain for 30-60 seconds. It was subsequently stained with Lugol's iodine for 30-60 seconds after which it was washed off with clean water. It was then decolorized rapidly in few seconds by dropping acetone until no more dye runs off from the smear. The smear was again stained with neutral red for 2 minutes and was quickly washed off and air dried. The preparation was then mounted on microscope and viewed using x10 and $\mathrm{x} 40$ objectives respectively.

\section{Results}

The result of this study showed that out of $60 \mathrm{HIV}$ positive patients that were examined for Neisseria gonorrhoeae co-infection, 40 patients $(66.7 \%)$ were infected with Neisseria gonorrhoeae. The prevalence of the infection among different age groups is shown in table 1. Individuals within the active age recorded the highest prevalence rate. Information on the marital status of the study participants used to determine the relationship between the different statuses and the infection prevalence infection prevalence shows that single unmarried individuals are at the greatest risk of infection followed by divorced groups (table 2).

Table 1: prevalence of Neisseria gonorrhoeae among different age group

\begin{tabular}{|l|l|l|}
\hline Age & No. screened & No. infected \\
\hline $11-20$ & 2 & 2 \\
\hline $21-30$ & 18 & 18 \\
\hline $31-40$ & 14 & 14 \\
\hline 41 and above & 6 & 6 \\
\hline
\end{tabular}

Table 2: relationship between infection and marital status

\begin{tabular}{|l|l|}
\hline Marital status & No. infected \\
\hline Single & 21 \\
\hline Married & 3 \\
\hline Divorced & 16 \\
\hline
\end{tabular}

The percentage prevalence of Neisseria gonorrhoeae isolates among sexes is shown in table 3 . Females recorded the highest prevalence (40\%) while the least prevalence was seen in their male counterpart (26.7\%).

Table 3: percentage Prevalence of Neisseria gonorrhoeae among sexes

\begin{tabular}{|l|l|l|l|}
\hline Sex & Total No. screened & No. infected & \%age prevalence \\
\hline Male & 20 & 16 & $26.7 \%$ \\
\hline Female & 40 & 24 & $40 \%$ \\
\hline Total & 60 & 40 & $46.7 \%$ \\
\hline
\end{tabular}

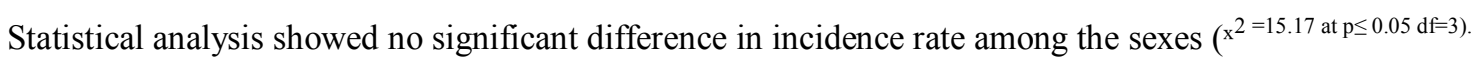




\section{Discussion}

Neisseria gonorrhoeae and other sexually transmitted diseases (STDs) are known to be associated in several ways with HIV. This is true because STDs and HIV are spread in like manner through sexual activities. People who engage in behaviours that encourages the spread of HIV are also more likely to contract STDs such gonorrhoeae (Wasserheit, 1992). Also, a person who contract HIV should be tested for other STDs. This study was carried out to investigate the possible co-infection of Neisseria gonorrhoeae in HIV positive patients. Out the 60 urine samples screened from the patients, $40(46.7 \%)$ had cases of gonorrhoeae. The rate of the infection was affected by the age distribution of the patients with those within the active age group (21-40) being at greatest risk of infection. This is in agreement with the earlier study carried out by Ene et al., (2007), who reported the prevalence of HIV and STDs infection in Nigeria to be highest among youths within the age group of 17-40. This is true because youthful age is the most active age and is therefore more prone to contracting sexually transmitted diseases. The relationship between marital status and infection prevalence as seen in this study shows that single -unmarried individuals and divorced groups who engage in indiscriminate sexual activities are the greatest risk of infection than their married counterpart. According Chesson, (2000), high STDs rate are found among women with heterosexual activities. High STDs rates such as gonorrhoeae, are associated with high HIV rates in some population of men who have sex with men (MSM), and may serve as predictors of an impending rise in HIV incidence (Torian, 2000). The prevalence of Neisseria gonorrhoeae among sexes recorded in this study shows that females have the highest incidence $(40 \%)$ than males with $26.7 \%$. This is evident because are more likely to engage in sexual activities than men. Sex workers are readily seen among women of different age groups and this behaviour predisposes them to sexually transmitted diseases such as gonorrhoeae more easily than their male counterparts. In addition, women genitals are more fragile and liable to develop ulcer which forms a vehicle for bacterial and viral transmission. Having an STD can make it easier to become infected with HIV and vice versa. This is due to various biological mechanisms. First, a person who has an STD that causes genital ulcers is more likely to contract HIV from an infected person because the open sores can serve as a portal for HIV to enter the body. Secondly, STDs may increase the risk of contracting HIV by affecting the integrity of cells. According to Hanson, (1998), persons with both ulcerative and non ulcerative STDs have a 2-5 times greater risk of becoming infected with HIV. Similarly, HIV positive women with STDs associated genital ulcers are more likely to transmit HIV and STD to their male partners than HIV positive women without such ulcers. In addition to the epidemiological pattern of STD and HIV transmission, immune damage related to HIV can influence the progression of STDs such as gonorrhoeae.

\section{Conclusion}

Because Of the Association between HIV and STDs many experts believe that integrated programs to prevent and manage the disease together are likely to be more effective than programs that treat HIV and STDs as separate problems. Community- wide interventions to detect and treat STDs may reduce the incidence of HIV in a population. In addition, a person who contracts an STD or HIV should be tested for both infections as infection with one could lead also pose a risk for the other.

\section{References}

[1] CDC, Guidelines for treatment of sexually transmitted diseases.
[2] Cheesbrough, M. District laboratory practical for tropical countries.

[3] Chesson, H. STDs and the increased risk for HIV transmissions: Implication for cost effectiveness analysis of STD prevention interventions. XIII International AIDs Conference, Durban South Africa, 2000, Abstract WePpCi321

[4] Ene, A.C., Ajayi, B.B., and Nwankwo, E.A. Prevalence of HIV Neonatal infection among babies born to HIV positive patients in Maiduguri, North Eastern Nigeria. Int J. Virol, 3(1), 2007, 41-44.

[5] Grosskurt, H. Control of sexually transmitted disease for HIV-1 prevention: Understanding the implication of the Mwanza and Rakai trials. Lancet 355, (9219), 2000, 1981.

[6] Hanson, J. Ulcerative and non ulcerative STD, increases HIV seroincidence in the U S: results from a longitudinal study in New Orleans, Louisiana. XIII International AIDs Conference, 1998, Abstract ThorC725.

[7] Ryan, K. Sherries medical microbiology ( $4^{\text {th }}$ Ed. Mc.Graw Hill, 2004).

[8] Torian, L. High HIV Seroprevalence associated with diagnosis of gonorrhoeae or syphilis in men who have sex with men. $7^{\text {th }}$ Conference on Retroviruses and Opportunistic Infections. 2000,Abstract, 468.

[9] Wasserheit, J.N. Epidemiological synergy: interrelationships between human immunodeficiency virus infection and other sexually transmitted diseases. Sexually transmitted diseases, 19,1992, 61-77. 